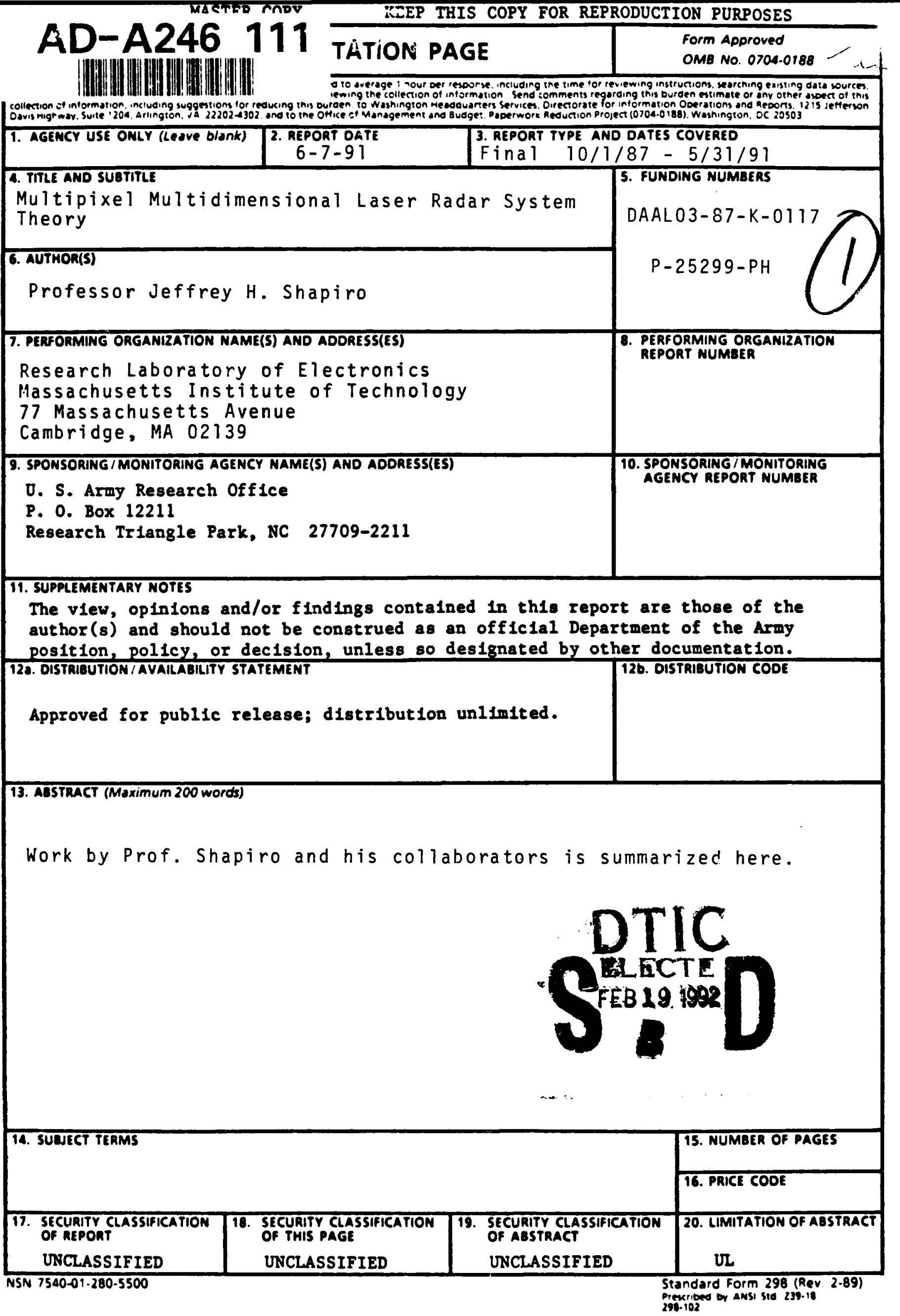




\section{MULTIPIXEL MULTIDIMENSIONAL LASER RADAR SYSTEM THEORY}

Final Report

Jeffrey $H$. Shapiro

Massachusetts Institute of Technology

U.S. Army Research Office Contract DAAL03-87-K-0117

Period Covered: $\quad 10 / 1 / 87-5 / 31 / 91$

Report Date: $\quad 6 / 7 / 91$ 


\section{Contents}

$\begin{array}{ll}\text { Abstract } & 2\end{array}$

Research Summary 3

Multipixel Multidimensional Target Detection . . . . . . . . . . . 4

Laser Radar Tracking Theory . . . . . . . . . . . . . . . 5

Synthetic Aperture Laser Radars . . . . . . . . . . . . . . 6

Adaptive Optics for Large-Aperture Laser Radars . . . . . . . . . . 6

Laser Radar Tomography . . . . . . . . . . . . . . . . . . 6

$\begin{array}{ll}\text { References } & 7\end{array}$

$\begin{array}{ll}\text { Personnel } & 10\end{array}$

$\begin{array}{ll}\text { Publications } & 11\end{array}$

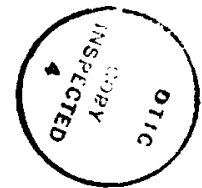

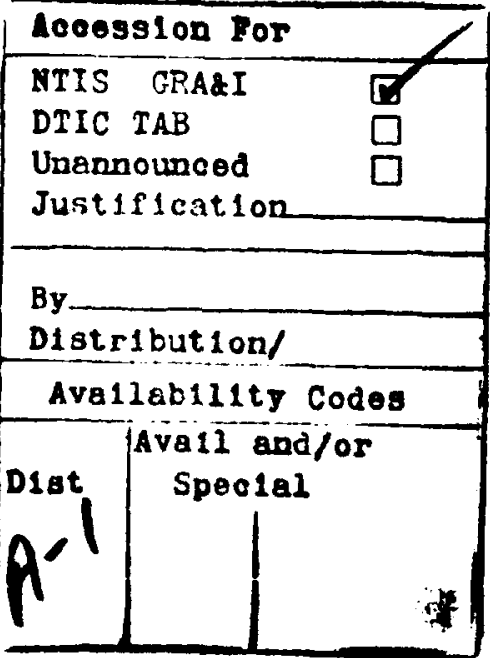




\section{MULTIPIXEL MULTIDIMENSIONAL LASER RADAR}

\section{SYSTEM THEORY}

\section{Abstract}

Coherent laser radars for tactical sensor applications are under development at

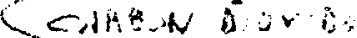
a number of laboratories, based on the mid-infrared technology of $\mathrm{CO}_{2}$ lasers and HgCdTe photodetectors. Test bed radars of this class have already demonstrated that tactical targets may be resolved in any combination of the modalities of space, angle, range, and velocity. Under U.S. Army Research Office Contract DAAL03-87-K-0117, a program of research was pursued to advance the system theory of such multipixel, multidimensional laser radars, and to corroborate these advances through experiments performed using the test bed coherent laser radars of the MIT Lincoln Laboratory Opto-Radar Systems Group. Major progress was made on the problem of target detection during the course of this work: quasi-optimal detection processors were derived for various 2-D and 3-D pulsed imagers; performance results for these processors were developed from computer simulation; and 2-D detection performance was confirmed experimentally. Substantial progress was also made on the problem of resolved-target tracking: a generalized Kalman filter was derived for linear-least $-s q u a r e s$ operation in a trackfwhile-image mode, with tracking performance studied both analytically and through simulation. Finally, a number of ancillary studies relating to laser speckle and atmospheric turbulence effects on laser radar performance were undertaken, including: theoretical treatments of synthetic aperture, range-Doppler, and tomographic laser radars; adaptive optics for large-aperture laser radars; and experimental verification of previous work on laser radar correlation scales. 


\section{Research Summary}

The development of laser technology offers new alternatives for the problems of target detection and imaging. Indeed, coherent laser radars based on the mid-infrared technology of $\mathrm{CO}_{2}$ lasers and $\mathrm{HgCdTe}$ photodetectors are under development at a number of laboratories [1]-[5]. Test bed radars of this class have already demonstrated that tactical targets may be resolved in any combination of the modalities of space, angle, range, and velocity. Until recently, however, the accompanying theory for coherent laser radar operation has only addressed single-pixel, single-dimensional issues relating to carrier-to-noise ratio (CNR), signal-to-noise ratio (SNR), and range or velocity measurement performance [6]-[11]. Under U.S. Army Research Office Contract DAAL03-87-K-0117, a program of research was pursued to advance the system theory of multipixel, multidimensional laser radars, and to corroborate these advances through experiments performed using the test bed coherent laser radars of the MIT Lincoln Laboratory Opto-Radar Systems Group.

The central foci for this program were determining the limits imposed by speckle and finite CNR on target detection and target tracking using multipixel, multidimensional, laser radar data. For 2-D pulsed-imager operation, quasi-optimal detection processors were derived, and associated performance results were obtained via computer simulation [12]-[14]. These studies produce the first quantitative assessment of the trade-offs between multidimensional data collection and detection performance, and their essential features were confirmed experimentally [14]. Quasi-optimal detection processors were also found for 3-D pulsed imagers, and computer simulation used to obtain their performance [15]. Experimental confirmation of the 3-D theory will be sought, along with processor and performance results for target recognition, 
which is an $M$-ary hypothesis testing task with $M>2$ [16]. In the target tracking area, a theory for track-while-image operation of a 2-D pulsed imager was developed, resulting in a generalized Kalman filter structure for linear-least-squares tracking [17]. Tracking performance was studied through Riccati equation evaluations and computer simulation [17],[18].

Additional theoretical studies were undertaken in the areas of synthetic aperture laser radar [19], adaptive optics for large-aperture laser radars [20], and laser radar tomography [21]. The latter two efforts were partially supported by the MIT Lincoln Laboratory Laser Radar Measurements Group. Also, an experimental verification of our earlier work on laser-radar speckle correlations [10] was performed, in cooperation with the Swedish Defense Research Establishment [22]. In what follows, we will survey the results obtained under U.S. Army Research Office Contract DAAL03-87-K-0117; for full details, the reader should consult the publications cited.

\section{Multipixel Multidimensional Target Detection}

Our initial efforts to establish the structure and performance of quasi-optimal, multipixel, multidimensional, target-detection processors [12]-[14] led to generalized likelihoodratio tests (GLRT's) for a full panoply of active-passive, 2-D pulsed-imager detection systems. The receiver operating characteristics (ROC's) for these processors were obtained through a combination of theoretical evaluation and computer simulation, and substantiated through experiments [14], using the MIT Lincoln Laboratory large aperture, multidimensional, laser radar test bed [23]. These studies employed realistic single-pixel statistical models for pulsed-imager intensity and range data, with the latter limited to range-resolution granularity. They assumed a downlooking detection scenario in which the presence or absence of a statistically-uniform, spatially-resolved, 
speckle target embedded in a statistically-uniform, spatially-resolved, speckle background is to be decided. Furthermore, the range and angular location of the target-if it is present within the image frame-as well as its reflectivity and that of the background, were treated as unknown parameters. A known planar background-range profile, and a range-unresolved target were assumed, however.

In more recent work, we have extended the preceding target-detection work in several ways [15],[16]. First, we removed the previous restriction to coarse-range (2-D) pulsed-imager operation, by introducing the correct statistical model for finerange (3-D) pulsed-imager operation. Second, we removed the restriction of known background-range profile, by using the estimation-maximization (EM) algorithm to do maximum-likelihood (ML) range estimation [16],[24]. Finally, we used a Markov random field (MRF) range processor [25] to seed the EM algorithm [15],[16]. This approach established a significant tie between MRF processing and statisticallyoptimum target detection, in that analog VLSI implementations of MRF processing are being proposed for target-recognition applications in next-generation radars.

\section{Laser Radar Tracking Theory}

Once a target has been detected, it frequently must be tracked. When an imaging laser radar is used to track a target, we have a multidpixel, multidimensional, multiframe system problem. In [17], we completed a first theoretical study of tracking an extended speckle target with a coherent laser radar. This work dealt with a target being tracked in the absence of background reflectors. It developed a generalized Kalman filter for linear-least-squares tracking in the track-while-image mode, and used Riccati equation evaluations to study tracking performance. In [18], we extended the approach used in [17] to account for the same downlooking background scenario employed in our 
target-detection work. This study, in addition to obtaining the tracker structure and theoretical performance, has used computer simulation to probe the loss-of-lock issue associated with image-frame boundaries.

\section{Synthetic Aperture Laser Radars}

We developed theoretical results for the spatial resolution and carrier-to-noise ratio behavior of the coherent laser radar version of the familiar microwave syntheticaperture radar (SAR) [19]. This work included the effects of target speckle, laser frequency instability, atmospheric turbulence, and radar/target motion effects. Sample system calculations showed that random vibrations were the most seriouis limitation on the use of SAR at optical frequencies, but such systems should be feasible with available laser frequency stabilities and typical turbulence strengths.

\section{Adaptive Optics for Large-Aperture Laser Radars}

We analyzed the use of wavelength-diversity adaptive optics to compensate for atmo spheric turbulence effects on a ground-based, large-aperture coherent laser radar [20]. We showed that as few as two wavelengths could provide very good discrimination of the turbulence-phase component from the speckle-phase component in the return from an extended target.

\section{Laser Radar Tomography}

We are completing an investigation [21] of the effects of target speckle on tomographic laser radar imaging [26]. This study comprises a thorough treatment of speckle and CNR limitations of backprojection and filtered backprojection tomographic reconstructions. 


\section{References}

[1] R.J. Hull and R.C. Harney, "Compact Infrared Radar Technology," Proc. SPIE 227, 162 (1980).

[2] M.J. Post, R.A. Richter, R.M. Hardesty, T.R. Lawrence, and F.F. Hall, Jr., "National Oceanic and Atmospheric Administration's (NOAA) Pulsed, Coherent, Infrared Doppler Lidar-Characteristics and Data," Proc. SPIE 300, 60 (1981).

[3] I. Renhorn, O. Steinvall, D. Letalick, K. Gullberg, T. Claesson, and A. Widén, "Performance Study of a Coherent Laser Radar," Proc. SPIE 415, 39 (1983).

[4] R. Foord, R. Jones, J.M. Vaughan, and D.V. Willets, "Precise Comparison of Experimental and Theoretical SNR's in $\mathrm{CO}_{2}$ Laser Heterodyne Systems," Appl. Opt. 22, 3787 (1983).

[5] J.Y. Wang, B.J. Bartholomew, M.L. Streiff, and E.F. Starr, "Imaging $\mathrm{CO}_{2}$ Laser Radar Field Tests," Appl. Opt. 23, 2565 (1984).

[6] J.H. Shapiro, B.A. Capron, and R.C. Harney, "Imaging and Target Detection with a Heterodyne-Reception Optical Radar," Appl. Opt. 20, 3292 (1981).

[7] J.H. Shapiro and S.T. Lau, "Turbulence Effects on Coherent Laser Radar Statistics," Appl. Opt. 21, 2395 (1982).

[8] J.H. Shapiro, "Target-Reflectivity Theory for Coherent Laser Radars," Appl. Opt. 21, 3398 (1982).

[9] D.M. Papurt, J.H. Shapiro, and S.T. Lau, "Measured Turbulence and Speckle Effects in Laser Radar Target Returns," Proc. SPIE 415, 166 (1983). 
[10] J.H. Shapiro, "The Correlation Scales of Laser Speckle in Heterodyne Detection," Appl. Opt. 24, 1883 (1985).

[11] J.H. Shapiro, R.W. Reinhold, and D. Park, "Performance Analyses for PeakDetecting Laser Radars," Proc. SPIE 663, 38 (1986).

[12] M.B. Mark and J.H. Shapiro, "Multipixel, Multidimensional Laser Radar System Performance," Proc. SPIE 783, 109 (1987).

[13] S.M. Hannon and J.H. Shapiro, "Laser Radar Target Detection with a Multipixel Joint Range-Intensity Processor," Proc. SPIE 999, 162 (1988).

[14] S.M. Hannon and J.H. Shapiro, "Active-Passive Detection of Multipixel Targets," Proc. SPIE 1222, 2 (1990).

[15] T.J. Green, Jr., J.H. Shapiro, and M.M. Menon, "Target Detection Performance Using 3-D Laser Radar Images, " Proc. SPIE 1471, in press.

[16] T.J. Green, Jr., "Three-Dimensional Object Detection and Recognition Using Multidimensional Laser Radar Imagery," Ph.D. thesis proposal, Dept. of Elect. Eng. and Comput. Sci., MIT, July 1991.

[17] R.H. Enders and J.H. Shapiro, "Laser Radar Tracking Theory," Proc. SPIE 999, 192 (1988).

[18] R.E. Mentle and J.H. Shapiro, "Track-While-Image in the Presence of Background," Proc. SPIE 1471, in press.

[19] D. Park and J.H. Shapiro, "Performance Analysis of Optical Synthetic Aperture Radars," Proc. SPIE 999, 100 (1988). 
[20] N.E. Zirkind and J.H. Shapiro, "Adaptive Optics for Large Aperture Coherent Laser Radars," Proc. SPIE 999, 117 (1988).

[21] B.T. Binder, "Laser Radar Tomography: The Effects of Speckle," Ph.D. thesis, Dept. of Elect. Eng. and Comput. Sci., MIT, August 1991.

[22] D. Letalick, I Renhorn, O Steinvall, and J.H. Shapiro, "Noise Sources in Laser Radar Systems," Appl. Opt. 28, 2657 (1989).

[23] D.U. Fluckiger, M.P. Bolduc, and L.A. Prior, "Large Aperture MultiDimensional Laser Radar Test Bed," Project Report FCT-5, MIT Lincoln Laboratory, 11 March 1988.

[24] J.H. Shapiro and T.J. Green, Jr., "Maximum-Likelihood Laser Radar Range Profling with the Estimation-Maximization Algorithm," in preparation.

[25] M.M. Menon, "Massively Parallel Image Restoration," Proc. SPIE 1471 in press.

[26] R.M. Marino, R.N. Capes, W.E. Keicher, S.R. Kulkarni, J.K. Parker, and L.W. Swezey, "Tomographic Image Reconstruction from Laser Radar Reflective Projections," Proc. SPIE 999, 248 (1988). 


\section{Personnel}

The research reported here was carried out by

Prof. Jeffrey H. Shapiro - Principal Investigator

Dr. Robert J. Hull

- Senior Investigator $(10 / 1 / 87-9 / 30 / 90)$

Dr. Paul J. Kolodzy

- Senior Investigator (10/1/90-5/31/91)

Dr. Dongwook Park

- Research Assistant (Ph.D. 1988)

Dr. Robert H. Enders

- Research Assistant (Ph.D. 1988)

Dr. Naomi E. Zirkind

- Graduate Student (Ph.D. 1989)

Dr. Stephen M. Hannon

- Research Assistant (Ph.D. 1989)

Mr. Robert E. Mentle

- Research Assistant (S.M. 1990)

Mr. Bradley T. Binder

- Graduate Student (Ph.D. expected 1991)

Mr. Thomas J. Green, Jr. - Research Assistant (Ph.D. expected 1992) 


\section{Publications}

The following journal articles, meeting papers, and theses have been produced under U.S. Army Research Office Contract DAAL03-87-K-0117.

1. D. Park, "High-Resolution Laser Radar Performance Analysis," Ph.D. thesis, Dept. of Elect. Eng. and Comput. Sci., MIT, January 1988.

2. R.H. Enders, "Laser Radar Tracking Theory," Ph.D.thesis, Dept. of Elect. Eng. and Comput. Sci., MIT, October, 1988.

3. D. Park and J.H. Shapiro, "Performance Analysis of Optical Synthetic Aperture Radars," Proc. SPIE 999, 100 (1988).

4. N.E. Zirkind and J.H. Shapiro, "Adaptive Optics for Large Aperture Coherent Laser Radars," Proc. SPIE 999, 117 (1988).

5. S.M. Hannon and J.H. Shapiro, "Laser Radar Target Detection with a Multipixel Joint Range-Intensity Processor, ${ }^{n}$ Proc. SPIE 999, 162 (1988).

6. R.H. Enders and J.H. Shapiro, "Laser Radar Tracking Theory," Proc. SPIE 999, 192 (1988).

7. N.E. Zirkind, "Adaptive Optics for Large Aperture Coherent Laser Radars," Ph.D. thesis, Dept. of Elect. Eng. and Comput. Sci., MIT, January 1989.

8. D. Letalick, I Renhorn, O Steinvall, and J.H. Shapiro, "Noise Sources in Laser Radar Systems," Appl. Opt. 28, 2657 (1989).

9. S.M. Hannon, "Detection Processing for Multidimensional Laser Radars," Ph.D. thesis, Dept. of Elect. Eng. and Comput. Sci., MIT, December 1989. 
10. S.M. Hannon and J.H. Shapiro, "Active-Passive Detection of Multipixel Targets, " Proc. SPIE 1222, 2 (1990).

11. R.E. Mentle, "Laser Radar Performance Theory for Track-While-Image Operation," S.M. thesis, Dept. of Elect. Eng. and Comput. Sci., MIT, June 1990.

12. T.J. Green, Jr., J.H. Shapiro, and M.M. Menon, "Target Detection Performance Using 3-D Laser Radar Images," Proc. SPIE 1471, in press.

13. R.E. Mentle and J.H. Shapiro, "Track-While-Image in the Presence of Background," Proc. SPIE 1471, in press.

14. B.T. Binder, "Laser Radar Tomography: The Effects of Speckle," Ph.D. thesis, Dept. of Elect. Eng. and Comput. Sci., MIT, August 1991.

15. T.J. Green, Jr., "Three-Dimensional Object Detection and Recognition Using Multidimensional Laser Radar Imagery," Ph.D. thesis proposal, Dept. of Elect. Eng. and Comput. Sci., MIT, July 1991.

16. J.H. Shapiro and T.J. Green, Jr., "Maximum-Likelihood Laser Radar Range Profiling with the Estimation-Maximization Algorithm," in preparation. 